\title{
Endocarditis in melioidosis
}

\section{B Piyasiri ${ }^{1}$, S A Wickramasinghe ${ }^{1}$, V C Lekamvasam ${ }^{1}$, E M Corea ${ }^{2}$, R Gunarathne $^{1}$, U Priyadarshana ${ }^{1}$}

Ceylon Medical Journal 2016; 61: 192-193

http://doi.org/10.4038/cmj.v61i4.8390

\section{Introduction}

Melioidosis, caused by Burkholderia pseudomallei, is a potentially fatal, emerging infection in Sri Lanka $[1,2]$. Although B.pseudomallei is known to infect almost every organ and system of the human body, infection of the heart valves is rare $[3,4]$. Here we report a case of melioidosis presenting as native valve endocarditis.

\section{Case report}

A 73 year old, male ex-smoker from Galle with well controlled chronic obstructive pulmonary disease (COPD), presented in September 2015 with anorexia and weight loss of 3 weeks duration and fever, cough and dyspnoea for 4 days. Patient was a paddy and chena cultivator.

On examination he was emaciated, pale, looked ill and was mildly dyspnoeic. He had a high volume pulse of $72 / \mathrm{bpm}$, a pan-systolic murmur in the mitral area with a loud pulmonary component of the second heart sound and bilateral basal crackles and rhonchi. Investigations showed a total white cell count of $27.8 \times 10^{9} / 1$ with neutrophilia (90\%), a platelet count of $208 \times 10^{9} / 1$, haemoglobin $9.2 \mathrm{~g} / \mathrm{dl}, \mathrm{CRP} 49 \mathrm{~g} / 1$, ESR $43 \mathrm{~mm} / 1 \mathrm{st}$ hour and normal blood sugar, renal and liver profiles. Blood picture revealed normochromic normocytic anaemia. His chest radiograph showed hyper-inflated lung fields with patchy consolidation. Ultrasound examination of the abdomen was normal. A provisional diagnosis of com-munity acquired pneumonia complicating COPD was made and the patient was started on intravenous cefotaxime and clarithromycin after taking blood and sputum for culture.

Blood culture grew an oxidase-positive Gramnegative bacillus after 27 hours of incubation and was reported as Pseudomonas spp. As chest infection with Pseudomonas is not uncommon in patients with COPD, he was treated with intravenous ceftazidime and ciprofloxacin according to the antibiotic sensitivity (ABST) report. However, since the clinical response was poor, a $2 \mathrm{D}$ echocardiogram was performed which showed mitral and aortic valve regurgitation and multiple small vegetations $(1 \times 1 \mathrm{~mm})$ on the aortic valve (Figure 1). The same antibiotics were continued for four weeks and, following improvement of clinical features, he was discharged on oral ciprofloxacin and oral cefixime.

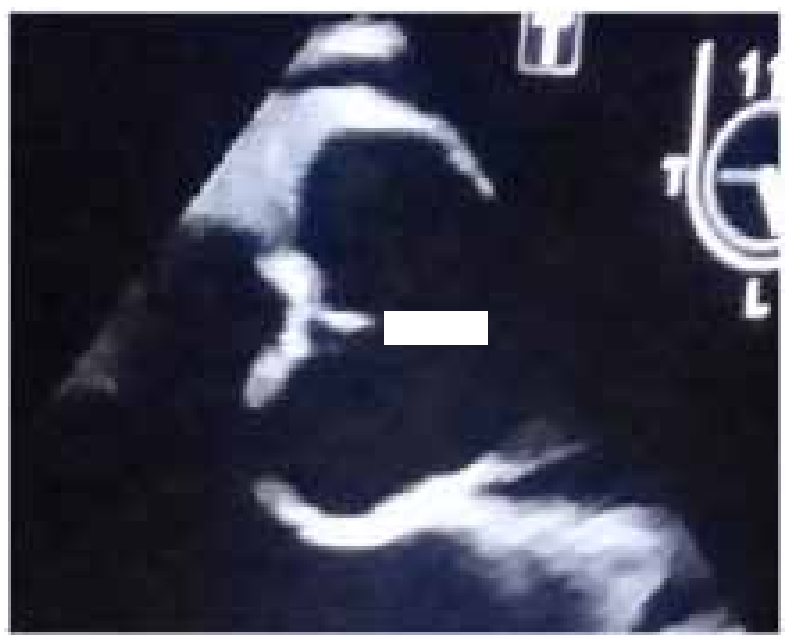

Figure 1. Trans-thoracic echocardiogram showing the vegetation attached to the aortic valve (arrow).

With the diagnosis of infective endocarditis, the blood culture bottles were traced and a fresh attempt was made to confirm the identity of the isolate. B. pseudomallei was suspected by the typical colony appearance and ABST and subsequently confirmed by PCR amplification of the LpxO gene. Patient's antibodies to B. pseudomallei by the indirect haemagglutination (IHA) test were only marginally positive at a titre of 40 . After discharge the patient had deteriorated clinically with increased CRP $(58 \mathrm{~g} / \mathrm{l}), \operatorname{ESR}(63 \mathrm{~mm} / \mathrm{hr})$ and vegetation size $(1 \times 3 \mathrm{~mm})$. He was readmitted and high dose intra-venous ceftazidime was started but this was switched to intravenous imipenem and oral cotrimoxazole later. The patient made a good recovery and CRP and ESR gradually returned to normal.

${ }^{1}$ Teaching Hospital, Karapitiya and 2Department of Microbiology, Faculty of Medicine, University of Colombo, Sri Lanka.

Correspondence: LBP, e-mail: <bhagyapiyasiri@yahoo.co.uk>. Received 29 April 2016 and revised version accepted 26 August 2016.

This is an open-access article distributed under the terms of the Creative Commons Attribution License, which permits unrestricted use, distribution, and reproduction in any medium, provided the original author and source are credited. 


\section{Discussion}

The clinical presentation of acute melioidosis can range from sepsis, pneumonia or deep seated abscesses of any organ of the human body to wound infections and cellulitis [4]. Infection is acquired from the environment through inoculation or inhalation and can remain latent and manifest many years later due to underlying risk factors like poor glycaemic control, immunosuppression [4]. Although the bacterium is known to infect any organ system of the human body, endocarditis is extremely rare $[3,4]$. Only a few cases have been reported worldwide and no cases from Sri Lanka could be found after an extensive literature search.

Melioidosis is difficult to diagnose clinically due to its protean manifestations. A history of occupational exposure to soil, as seen in our patient, may be useful. Infection is more common in diabetics but is not restricted to them, as illustrated by our patient whose risk factor was COPD. In view of the emergence of melioidosis in Sri Lanka, clinicians should have a high index of suspicion in at risk populations. As many medical laboratory technicians are unfamiliar with the colony appearance of $B$. pseudomallei, it may be misinterpreted as Pseudomonas, as in our case. Adequate training and the resources should be available for accurate identification [1]. Although antibody titres are usually $>320$ in culture positive cases, low titres, as seen in our patient, do not exclude melioidosis.

Treatment of the acute phase of melioidosis involves the use of ceftazidime or a carbapenem, with or without cotrimoxazole, for at least two weeks. After 2-3 weeks of intravenous therapy, an oral regime consisting of doxy- cycline or cotrimoxazole is used for eradication therapy for a minimum of three more months [5]. However, it is likely that a patient with endocarditis will need a longer duration of intravenous antibiotic therapy and a longer duration of oral therapy and close monitoring with longterm follow up.

\section{Conflicts of interest}

There are no conflicts of interest.

\section{References}

1. Corea EM, Merritt AJ, Ler YH, Thevanesam V, Inglis TJ. Sri Lankan National Melioidosis Surveillance Program Uncovers a Nationwide Distribution of Invasive Melioidosis Am J Trop Med Hyg 2016; 94: 292-8.

2. Corea E, Thevanesam V, Perera S, et al. Melioidosis in Sri Lanka: an emerging infection. Sri Lankan J Infect Dis 2012; 2: $2-8$.

3. Mansoor CA, Jemshad A, Meliodosis with endocarditis and massive cerebral infarct. Ital J Med, doi:10.4081/ itjm.2015.589

4. Punyagupta S. Melioidosis, Review of 686 Cases and Presentation of A New Clinical Classification, Melioidosis, Punyagupta S, Sirisanthana T, Stapatayavong B (Eds), Bangkok Medical Publisher, Bangkok 1989: 217-29.

5. Inglis TJ. The treatment of melioidosis. Pharmaceuticals 2010; 3: 1296-303. 